\title{
Treatment of resistant metastatic melanoma using sequential epigenetic therapy (decitabine and panobinostat) combined with chemotherapy (temozolomide)
}

\author{
Chang Xia $\cdot$ Roberto Leon-Ferre $\cdot$ Douglas Laux $\cdot$ \\ Jeremy Deutsch • Brian J. Smith • Melanie Frees • \\ Mohammed Milhem
}

Received: 8 December 2013 / Accepted: 27 May 2014 / Published online: 26 July 2014

(C) The Author(s) 2014. This article is published with open access at Springerlink.com

\begin{abstract}
Purpose To explore the safety and tolerability of combining two epigenetic drugs: decitabine (a DNA methyltransferase inhibitor) and panobinostat (a histone deacetylase inhibitor), with chemotherapy with temozolomide (an alkylating agent). The purpose of such combination is to evaluate the use of epigenetic priming to overcome resistance of melanoma to chemotherapy.

Methods A Phase I clinical trial enrolling patients aged 18 years or older, with recurrent or unresectable stage III or IV melanoma of any site. This trial was conducted with full Institutional Review Board approval and was
\end{abstract}

C. Xia $\cdot$ M. Frees $\cdot$ M. Milhem $(\bowtie)$

Division of Hematology, Oncology and Bone and Marrow

Transplantation, Department of Internal Medicine, University

of Iowa Hospitals and Clinics, 200 Hawkins Drive, Iowa City,

IA 52242, USA

e-mail: mohammed-milhem@uiowa.edu

R. Leon-Ferre

Department of Internal Medicine, University of Iowa Hospitals and Clinics, 200 Hawkins Drive, Iowa City, IA 52242, USA

D. Laux

Oncology-Hematology, St Mary's Health Center, Richmond

Heights, MO, USA

D. Laux

Oncology-Hematology, Capital Region Medical Center, 1241 W

Stadium Blv, Jefferson City, MO 65109, USA

J. Deutsch

Oncology-Hematology, Cancer Center of Kansas, $2322 \mathrm{~N}$

Chelmsford, Wichita, KS 67228, USA

B. J. Smith

Department of Biostatistics, College of Public Health, University

of Iowa, 105 River St, Iowa City, IA 52242, USA registered with the National Institutes of Health under the clinicaltrials.gov identifier NCT00925132. Patients were treated with subcutaneous decitabine 0.1 or $0.2 \mathrm{mg} /$ $\mathrm{kg}$ three times weekly for 2 weeks (starting on day 1), in combination with oral panobinostat 10,20 , or $30 \mathrm{mg}$ every $96 \mathrm{~h}$ (starting on day 8 ), and oral temozolomide $150 \mathrm{mg} / \mathrm{m}^{2} /$ day on days 9 through 13 . In cycle 2 , temozolomide was increased to $200 \mathrm{mg} / \mathrm{m}^{2} /$ day if neutropenia or thrombocytopenia had not occurred. Each cycle lasted 6 weeks, and patients could receive up to six cycles. Patients who did not demonstrate disease progression were eligible to enter a maintenance protocol with combination of weekly panobinostat and thrice-weekly decitabine until tumor progression, unacceptable toxicity, or withdrawal of consent.

Results Twenty patients were initially enrolled, with 17 receiving treatment. The median age was 56 years. Eleven $(65 \%)$ were male, and $6(35 \%)$ were female. Eleven $(64.7 \%)$ had cutaneous melanoma, $4(23.5 \%)$ had ocular melanoma, and $2(11.8 \%)$ had mucosal melanoma. All patients received at least one treatment cycle and were evaluable for toxicity. Patients received a median of two 6-week treatment cycles (range 1-6). None of the patients experienced DLT. MTD was not reached. Adverse events attributed to treatment included grade 3 lymphopenia (24\%), anemia (12\%), neutropenia (12\%), and fatigue $(12 \%)$, as well as grade 2 leukopenia (30\%), neutropenia $(23 \%)$, nausea $(23 \%)$, and lymphopenia (18\%). The most common reason for study discontinuation was disease progression.

Conclusions This triple agent of dual epigenetic therapy in combination with traditional chemotherapy was generally well tolerated by the cohort and appeared safe to be continued in a Phase II trial. No DLTs were observed, and MTD was not reached. 
Keywords Melanoma $\cdot$ Epigenetics $\cdot$ Epigenetic priming $\cdot$ Resistance $\cdot$ Hypomethylation $\cdot$ Histone deacetylation

\section{Introduction}

Until the recent advances in immune and targeted therapeutic approaches, progress in the treatment of metastatic melanoma remained dormant for nearly two decades. The approval of the immune stimulant ipilimumab and the subsequent development of novel targeted agents against BRAF, MEK, and PD-1 have fundamentally changed the landscape of melanoma treatment. Despite the excitement generated by these novel agents, much remains to be understood and significant hurdles remain to be conquered. When individual oncogenic pathways are blocked pharmacologically, melanoma cells find ways to adapt and selectively activate alternative pathways that allow them to "escape" the effects of targeted agents. To prevent this, various trials are evaluating the combined use of drugs targeting multiple pathways simultaneously. While targeting multiple downstream effectors of these pathways might be beneficial, we believe that depriving the cells of the ability to adapt and selectively activate such pathways by targeting upstream epigenetic mechanisms might be a more effective approach.

Epigenetic manipulation is a novel approach to cancer therapy that has proven successful in the treatment of hematologic malignancies, but remains to be further explored in solid tumors. Epigenetic alterations contribute to melanomagenesis by down-regulating tumor suppressor genes, apoptotic mediators, and DNA repair enzymes [1]. They also appear to be an important driving force in resistance mechanisms to multiple therapies. There is evidence that epigenetic silencing may contribute to resistance to chemotherapeutics and that drugs targeting epigenetic mechanisms may enhance chemosensitivity [2, 3]. Epigenetic drugs also appear to enhance the endogenous antitumor immune response via several mechanisms including, but not limited to, increased expression of cancer-testis antigens [4-14]. Furthermore, epigenetic drugs have shown the ability of reconstituting the functionality of apoptotic processes that, when deregulated, appear to play a crucial role in the resistance to chemotherapeutics [15], immune responses [11, 16], and targeted agents such as BRAF and MEK inhibitors [17, 18]. These, along with many other potential mechanisms, support the notion that epigenetic modifications represent a global mechanism for treatment resistance in melanoma.

In this Phase I trial, we explore the safety and tolerability of combining two epigenetic drugs: decitabine [a DNA methyltransferase (DNMT) inhibitor] and panobinostat [a
Table 1 Doses of decitabine and panobinostat ${ }^{\mathrm{a}}$

\begin{tabular}{llll}
\hline Cohort & $\begin{array}{l}\text { Decitabine (subcutaneously, } \\
\text { three times weekly for } \\
\text { 2 weeks) }(\mathrm{mg} / \mathrm{kg})\end{array}$ & $\begin{array}{l}\text { Panobinostat } \\
\text { (orally, every } \\
96 \mathrm{~h})(\mathrm{mg})\end{array}$ & No. of patients \\
\hline 1 & 0.1 & 10 & 5 \\
2 & 0.1 & 20 & 4 \\
3 & 0.2 & 20 & 4 \\
4 & 0.2 & 30 & 4 \\
\hline
\end{tabular}

a All cohorts received oral temozolomide at a dose of $150 \mathrm{mg} / \mathrm{m}^{2} /$ day on days 9 through 13 on cycle 1

histone deacetylase (HDAC) inhibitor], with traditional chemotherapy with temozolomide (an alkylating agent), setting the stage of epigenetic interruption of melanoma cell resistance. This trial started enrolling patients when temozolomide was a standard treatment for metastatic melanoma, prior to the approval of ipilimumab and subsequent targeted therapies. The primary objective of this trial was to evaluate the safety and tolerability of this triple agent regimen at previously defined doses. Since the use of decitabine in this trial was aimed at achieving epigenetic modification and not cytotoxicity, decitabine was administered at low doses known to cause hypomethylation. Panobinostat was dose-escalated as shown in Table 1. Temozolomide was administered at standard doses. While our model tested epigenetic drugs in combination with chemotherapy, we believe that a similar approach could be used with the newer immune and targeted therapies.

\section{Materials and methods}

Patients and eligibility criteria

Eligible participants included male or female patients that were 18 years of age or older, with recurrent or unresectable stage III or IV melanoma of any site. Since we sought to evaluate enhancement of chemosensitivity by epigenetic drugs, this trial enrolled patients with inherently aggressive and resistant disease, including noncutaneous melanoma like ocular and mucosal; patients with brain metastases, after the brain disease was adequately addressed either by whole brain radiation, radiosurgery, or resection; and patients that had progressed during or after their most recent treatment. Eligibility criteria also included adequate liver, renal, cardiac and bone marrow function; normal electrolytes; normal thyroid function (or on adequate replacement doses); normal LVEF by MUGA or echocardiogram; measurable disease per RECIST 1.0 criteria; and Eastern Cooperative Oncology Group (ECOG) performance status of 0-2. Previously treated or treatment-naïve patients were both eligible, except those who had previously received 
valproic acid, HSP90 inhibitors, hypomethylating agents, or HDAC inhibitors. Female patients of childbearing potential were required to not be pregnant, breast-feeding, and to use double contraception during and 3 months after study completion.

Exclusion criteria included: uncontrolled hypertension; history of ventricular fibrillation, torsades de pointes, or sustained ventricular tachycardia; heart rate $<50$ beats/ min; congestive heart failure NYHA class III or IV; acute coronary syndrome within 6 months of study enrollment; ECG abnormalities of QTc prolongation ( $>450 \mathrm{~ms}$ ), right bundle branch block or left anterior hemiblock; known HIV or hepatitis C positivity; unresolved diarrhea or significant gastrointestinal impairment potentially interfering with panobinostat or temozolomide absorption; and concomitant use of CYP3A4 inhibitors or drugs known to increase risk of torsades de pointes.

This trial was conducted with full Institutional Review Board approval. All participants provided written consent before participating. This study was registered with the National Institutes of Health under the clinicaltrials.gov identifier NCT00925132. Novartis provided panobinostat and financial support for this trial.

\section{Study treatment and dose escalation}

Patients were treated with subcutaneous decitabine at a dose of 0.1 or $0.2 \mathrm{mg} / \mathrm{kg}$ three times weekly for 2 weeks (starting on day 1), in combination with oral panobinostat at a dose of 10,20 , or $30 \mathrm{mg}$ every $96 \mathrm{~h}$ (starting on day 8 ), and oral temozolomide at a dose of $150 \mathrm{mg} / \mathrm{m}^{2} /$ day on days 9 through 13 (Fig. 1; Table 1). In cycle 2, temozolomide dose was increased to $200 \mathrm{mg} / \mathrm{m}^{2} /$ day if neutropenia or thrombocytopenia had not occurred. Prophylactic trimethoprim-sulfamethoxazole was not used. Each treatment cycle lasted 6 weeks, and patients could receive up to six cycles of combination treatment. Patients who did not demonstrate disease progression were eligible to enter a maintenance protocol with combination of weekly panobinostat and thrice-weekly decitabine until tumor progression, unacceptable toxicity, or withdrawal of consent. Maximum tolerated dose (MTD) was defined as the highest dose cohort where $\leq 1 / 6$ patients experienced a dose-limiting toxicity
(DLT). If a DLT was observed in the first three patients, the cohort was expanded to six patients, and all six patients needed to complete the first cycle of therapy without an additional DLT before dose escalation could proceed. Intrapatient dose escalation was not allowed. DLT was defined as grade 4 hematologic toxicity, grade $\geq 3$ nonhematologic toxicity, or grade 2 nonhematologic or grade 3 hematologic toxicity requiring a dose reduction or treatment interruption for more than 7 days during the first cycle. Grade 3 or 4 nausea, vomiting, or diarrhea were only considered DLTs if they occurred despite optimal medical management. Grade 3 electrolyte, uric acid, or phosphorus abnormalities were not considered DLTs if they were correctable within 1 week.

In cycle 2 , dose was increased to $200 \mathrm{mg} / \mathrm{m}^{2} /$ day if there was no neutropenia or thrombocytopenia.

Safety and response assessments

Patients were assessed for safety every 2 weeks during the first two cycles and then once every cycle. $\mathrm{CBC}$ with differential and serum chemistries were obtained once a week during the first two cycles and every 2 weeks thereafter. ECGs were performed prior to and following the first dose of panobinostat during cycle 1 , and then on day 8 of every subsequent cycle. Toxicity was graded according to the National Cancer Institute (NCI) Common Terminology Criteria for Adverse Events (CTCAE), version 3.0.

Tumor response was assessed using whole body FDG PET-CT or CT scan after two cycles of treatment. Response was determined based on the response evaluation criteria in solid tumors (RECIST).

\section{Results}

Patient characteristics

Twenty patients were enrolled in the Phase I portion of this study. One patient had rapid progression of disease and was not treated with the protocol. One patient did not meet eligibility criteria due to untreated brain metastases. One patient withdrew consent. A total of seventeen patients

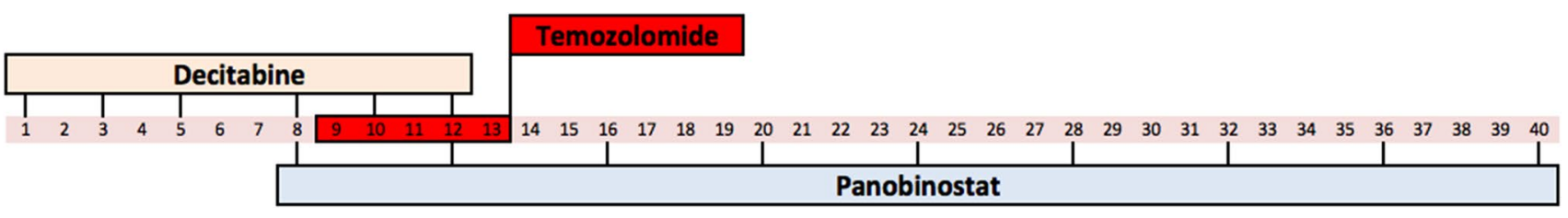

Fig. 1 Treatment schema. Cycle duration: 42 days. Decitabine: days 1, 3, 5, 8, 10, 12. Panobinostat: days 8, 12, 16, 20, 24, 28, 32, 36, 40. Temozolomide: days 9-13 
Table 2 Patient characteristics $(n=17)$

\begin{tabular}{ll}
\hline Characteristics & Value (range) \\
\hline Male:female & $11: 6$ \\
Median age & $56(32-77)$ \\
Melanoma location & \\
Cutaneous & 11 \\
Ocular & 4 \\
Mucosal & 2 \\
Median no. of prior systemic treatments & $1(0-3)$ \\
ECOG & $0-1$ \\
Median no. of cycles administered & $2(1-6)$ \\
\hline
\end{tabular}

received treatment. Characteristics are listed in Table 2. The median age was 56 years. Eleven $(65 \%)$ were male, and $6(35 \%)$ were female. Eleven $(64.7 \%)$ had cutaneous melanoma, $4(23.5 \%)$ had ocular melanoma, and 2 $(11.8 \%)$ had mucosal melanoma.

\section{Exposure to treatment and clinical toxicities}

All seventeen patients received at least one treatment cycle and were evaluable for toxicity. Patients received a median of two 6-week treatment cycles (range 1-6). The dose escalation schema and number of patients enrolled in each cohort are described in Table 1 . None of the patients experienced DLT. One patient in cohort 3 had grade 4 neutropenia that resolved within 3 days and did not meet criteria to be categorized as DLT. MTD was not achieved. Adverse events in each cohort are summarized in Table 3. These included grade 3 lymphopenia (24\%), anemia (12\%), neutropenia (12\%), and fatigue $(12 \%)$, as well as grade 2 leukopenia (30\%), neutropenia (23\%), nausea (23\%), and lymphopenia $(18 \%)$. The most common reason for study discontinuation was disease progression.

The majority of the adverse events occurred in cohort 3 . Of the 10 adverse events observed, only 8 were deemed to be treatment related (hypokalemia and back pain were not treatment related). Subjects' characteristics and underlying disease might have contributed to the relatively high occurrence of adverse events in Cohort 3. Two out of the four subjects in cohort 3 were withdrawn from the study due to rapid disease progression and died shortly after.

\section{Treatment efficacy}

Of the 17 patients treated, 9 were considered nonevaluable for efficacy given that they did not complete two cycles of therapy due to early disease progression. Among eight patients evaluable for radiographic response, $6(75 \%)$ had either stable disease $(5,62.5 \%)$ or complete response $(1,12.5 \%)$. The patient with the complete response had mucosal melanoma and had the best response after two cycles of therapy. Response lasted for 8 months. Of the five patients with stable disease, two were from Cohort 1, two from Cohort 3, and one from Cohort 4. The two remaining patients $(25 \%)$ had progressive disease after two cycles of therapy (Table 4).

\section{Discussion}

The field of epigenetics might offer a novel approach to the treatment of melanoma that could potentially add to the recent progress in immune and targeted therapies. Though
Table 3 Summary of adverse events

\footnotetext{
${ }^{a}$ No. of subjects: number of subjects that developed the adverse event listed

b Subject that developed each adverse event is listed to note that some of the adverse events occurred in the same subject in a given cohort
}

\begin{tabular}{|c|c|c|c|c|c|c|c|}
\hline Cohort & $\begin{array}{l}\text { Grade } 3 \text { (no. of } \\
\text { subjects) }^{\mathrm{a}}\end{array}$ & Subject $^{\mathrm{b}}$ & $\begin{array}{l}\text { Cycle } \\
\text { and day }\end{array}$ & $\begin{array}{l}\text { Grade } 4 \\
\text { (no. of subjects) }\end{array}$ & Subject $^{\mathrm{b}}$ & $\begin{array}{l}\text { Cycle } \\
\text { and day }\end{array}$ & DLT \\
\hline \multirow[t]{4}{*}{1} & Lymphopenia (1) & \#03 & $\mathrm{C} 1, \mathrm{D} 12$ & None & & & None \\
\hline & Anemia (1) & \#02 & $\mathrm{C} 1, \mathrm{D} 12$ & & & & \\
\hline & Fatigue (1) & $\# 02$ & C2, D3 & & & & \\
\hline & Nausea (1) & $\# 02$ & $\mathrm{C} 2, \mathrm{D} 3$ & & & & \\
\hline 2 & None & & & None & & & None \\
\hline \multirow[t]{9}{*}{3} & Lymphopenia (3) & $\# 12$ & $\mathrm{C} 1, \mathrm{D} 40$ & Neutropenia (1) & $\# 13$ & $\mathrm{C} 1, \mathrm{D} 21$ & None \\
\hline & Anemia (1) & $\# 13$ & $\mathrm{C} 1, \mathrm{D} 40$ & & & & \\
\hline & Neutropenia (1) & $\# 14$ & $\mathrm{C} 1, \mathrm{D} 15$ & & & & \\
\hline & Fatigue (1) & $\# 14$ & $\mathrm{C} 1, \mathrm{D} 15$ & & & & \\
\hline & Fever (1) & $\# 14$ & $\mathrm{C} 1, \mathrm{D} 20$ & & & & \\
\hline & Hypokalemia (1) & $\# 15$ & C4, D18 & & & & \\
\hline & Back pain (1) & $\# 15$ & $\mathrm{C} 2, \mathrm{D} 3$ & & & & \\
\hline & & $\# 15$ & C3, D5 & & & & \\
\hline & & $\# 12$ & C1, D33 & & & & \\
\hline 4 & Thrombocytopenia (1) & \#20 & $\mathrm{C} 1, \mathrm{D} 25$ & None & & & None \\
\hline
\end{tabular}


Table 4 Response assessment (by RECIST criteria)

\begin{tabular}{lll}
\hline Cohort & Melanoma origin & Type of response \\
\hline 1 & Cutaneous & Stable disease \\
1 & Cutaneous & Stable disease \\
1 & Mucosal & Complete response \\
2 & Ocular & Progression \\
3 & Ocular & Stable disease \\
3 & Cutaneous & Stable disease \\
4 & Mucosal & Stable disease \\
4 & Ocular & Progression \\
\hline
\end{tabular}

significantly explored in hematologic malignancies, epigenetic therapeutic approaches have lagged behind in solid tumors. It has been well established that epigenetic processes, specifically DNA promoter methylation and histone acetylation/deacetylation, are key cellular events during tumorigenesis [19]. Moreover, melanoma cells appear to use the epigenetic apparatus to "adapt" and acquire resistance to external offenders such as chemotherapeutics [15], the immune system $[11,16]$, and even the newer targeted agents $[17,18]$. In this Phase I trial, we explored the safety and tolerability of traditional chemotherapy combined with dual epigenetic therapy with sequential DNMT and HDAC inhibition. We used temozolomide, a known standard agent for metastatic melanoma prior to 2011, combined with decitabine and panobinostat.

During DNA methylation, a methyl group is added to cytosine in $\mathrm{CpG}$ islands located predominantly in promoter regions, resulting in the silencing of genes regulated by the affected promoter. Abnormal genetic silencing by DNA methylation appears to modulate cancer biology and development of drug resistance [20]. Decitabine is a powerful DNMT inhibitor that has shown the ability to impair the methylation process in numerous cancer cell lines (including melanoma), allowing the re-expression of genes that malignant cells are trying to turn off [5]. There is evidence that the doses required to achieve hypomethylation are much lower than the usual cytotoxic doses [21-23]. In addition, given that active cell cycling is required to achieve methylation reversal, prolonged courses achieve more hypomethylation than shorter courses [24]. In myeloid neoplasms, extended administration of low doses of hypomethylating agents may result in increased or sustained response rates [25]. When the goal is to achieve and maintain methylation reversal, keeping decitabine toxicity to a minimum might be key, as this allows for repeated doses and longer courses. This approach might be more effective than trying to push decitabine doses until DLT or MTD are reached [20]. Most trials using decitabine in solid tumors have used high, toxic doses, with short administration periods [26-29]. These factors, at least partly, may account for the disappointing responses to decitabine in solid tumors, as compared to responses in hematopoietic malignancies. For these reasons, in this trial, we used low doses of decitabine in an extended dosing regimen. DLT or MTD were not reached, allowing for repeated dose administration.

In addition to promoter methylation patterns, gene expression is also highly influenced by DNA-histone interactions that regulate the ability of the transcription apparatus to access the DNA. The opposing activities of histone acetyltransferase and HDAC maintain histone acetylation patterns that lead to cell-specific gene expression profiles. Aberrant HDAC recruitment appears to play a critical role in gene expression changes seen in malignant transformed cells that allow them to block apoptotic mechanisms. HDAC inhibitors appear to reestablish apoptosis in melanoma cells [30], induce cell differentiation, and inhibit tumor growth in animal models by down-regulating positive cell cycle regulators such as cyclin D1, c-Myc, C-RAF, and AKT [31-37], while inducing the expression of a number of anti-proliferative genes [38-40]. Melanoma cells exposed to HDAC inhibitors also exhibit decreased levels of activated MEK1/2 and ERK1/2 [41], key melanomagenic kinases blocked by novel targeted agents. HDAC inhibitors may also interfere with the appropriate folding of HSP90-client proteins (including AKT and RAF) that are critical to cancer cell growth [36, 37]. HDAC inhibitors, however, are unable to reactivate the expression of genes that have been previously silenced by methylation of their promoters. This provides a rationale for the sequential use of DNMT inhibitors followed by HDAC inhibitors to provide "epigenetic synergy," which has shown to enhance gene re-expression and drug sensitivity [42]. In this trial, we administered Panobinostat, a novel and potent HDAC inhibitor, a week after decitabine initiation. In animal studies, panobinostat has been shown to have affinity to melanin, judged by measurable drug-related radioactivity in the uveal and pigmented skin at $96 \mathrm{~h}$ port-dose administration (data from Novartis Investigator's Brochure). In our study, panobinostat was administered every $96 \mathrm{~h}$.

This triple agent regimen of decitabine, panobinostat, and temozolomide was generally well tolerated by the cohort and appeared safe to be continued in a Phase II trial. No DLTs were observed, and MTD was not reached. As discussed before, when the goal is to achieve epigenetic modulation, administration of higher doses of DNMT inhibitors might hinder the ability of patients to tolerate the frequent dosing intervals required to maintain hypomethylation throughout the treatment cycle. Maintaining hypomethylation also appears to be more important than deepening the nadir of methylation in each cycle. Given that the doses used in all cohorts proved safe, cohort 3 dose level was the recommended dose for the Phase II portion of this trial, as this is the subcutaneous dose of decitabine that has 
been shown to achieve successful hypomethylation [43]. This is supported by the observation of a gradual increase of hemoglobin $\mathrm{F}$ concentration 2 weeks after the initiation of therapy (preliminary data from Phase II, not shown) that appears to persist through the course of treatment. Patient responses in this cohort as shown in Table 4 are intriguing, especially given the inclusion of patients with ocular and mucosal melanoma, two highly chemoresistant variants of melanoma. The complete response observed in one subject with mucosal melanoma is very intriguing. This subject achieved this best response (complete response) after two cycles. Mucosal melanomas tend to have c-kit mutations and are generally negative for B-RAF. They metastasize quite frequently, behaving differently from cutaneous melanoma. The effect of epigenetic therapy in this patient population might provide clues to the disease biology and warrants more investigation. However, the rarity of these tumors will likely make studying this subpopulation more difficult. We hypothesize that DNMT inhibition followed by HDAC inhibition target key epigenetic events that melanoma cells use to selectively turn on or off specific pathways that confer resistance to chemotherapy and apoptosis. This study started enrolling patients prior to the approval of ipilimumab and the new targeted agents that are revolutionizing the treatment of melanoma. We believe that epigenetic alterations might represent a global resistance mechanism in melanoma and other cancers. Clinical trials using a similar approach, but this time combining epigenetic agents with immune therapies and novel targeted agents such as BRAF, MEK, or PD-1 inhibitors, are warranted. This approach of crippling "upstream" epigenetic mechanisms that allow melanoma cells to adapt and acquire resistance to novel agents could prove to be an alternative to blocking multiple "downstream" effectors using multiple targeted agents.

Open Access This article is distributed under the terms of the Creative Commons Attribution License which permits any use, distribution, and reproduction in any medium, provided the original author(s) and the source are credited.

\section{References}

1. Alcazar O, Achberger S, Aldrich W, Hu Z, Negrotto S, Saunthararajah Y, Triozzi P (2012) Epigenetic regulation by decitabine of melanoma differentiation in vitro and in vivo. Int $\mathrm{J}$ Cancer 131(1):18-29. doi:10.1002/ijc.26320

2. Teodoridis JM, Hall J, Marsh S, Kannall HD, Smyth C, Curto J, Siddiqui N, Gabra H, McLeod HL, Strathdee G, Brown R (2005) CpG island methylation of DNA damage response genes in advanced ovarian cancer. Cancer Res 65(19):8961-8967. doi:10.1158/0008-5472.CAN-05-1187

3. Glasspool RM, Teodoridis JM, Brown R (2006) Epigenetics as a mechanism driving polygenic clinical drug resistance. Br J Cancer 94(8):1087-1092. doi:10.1038/sj.bjc.6603024
4. Dubovsky JA, McNeel DG (2007) Inducible expression of a prostate cancer-testis antigen, SSX-2, following treatment with a DNA methylation inhibitor. Prostate 67(16):1781-1790. doi:10.1002/pros.20665

5. Coral S, Sigalotti L, Covre A, Nicolay HJ, Natali PG, Maio M (2007) 5-AZA-2'-deoxycytidine in cancer immunotherapy: a mouse to man story. Cancer Res 67(6):2900-2901; author reply 2901-2902. doi:10.1158/0008-5472.CAN-06-2986

6. Guo ZS, Hong JA, Irvine KR, Chen GA, Spiess PJ, Liu Y, Zeng G, Wunderlich JR, Nguyen DM, Restifo NP, Schrump DS (2006) De novo induction of a cancer/testis antigen by 5-aza-2'-deoxycytidine augments adoptive immunotherapy in a murine tumor model. Cancer Res 66(2):1105-1113. doi:10.1158/0008-5472.CAN-05-3020

7. Grunau C, Sanchez C, Ehrlich M, van der Bruggen P, Hindermann W, Rodriguez C, Krieger S, Dubeau L, Fiala E, De Sario A (2005) Frequent DNA hypomethylation of human juxtacentromeric BAGE loci in cancer. Genes Chromosom Cancer 43(1):1124. doi:10.1002/gcc. 20155

8. Weber J, Salgaller M, Samid D, Johnson B, Herlyn M, Lassam N, Treisman J, Rosenberg SA (1994) Expression of the MAGE-1 tumor antigen is up-regulated by the demethylating agent 5-aza2'-deoxycytidine. Cancer Res 54(7):1766-1771

9. de Vos D, van Overveld W (2005) Decitabine: a historical review of the development of an epigenetic drug. Ann Hematol 84(Suppl 1):3-8. doi:10.1007/s00277-005-0008-x

10. Jaenisch R, Bird A (2003) Epigenetic regulation of gene expression: how the genome integrates intrinsic and environmental signals. Nat Genet 33(Suppl):245-254. doi:10.1038/ng1089

11. Konkankit VV, Kim W, Koya RC, Eskin A, Dam MA, Nelson S, Ribas A, Liau LM, Prins RM (2011) Decitabine immunosensitizes human gliomas to NY-ESO-1 specific T lymphocyte targeting through the Fas/Fas ligand pathway. J Transl Med 9:192. doi:10.1186/1479-5876-9-192

12. Almstedt M, Blagitko-Dorfs N, Duque-Afonso J, Karbach J, Pfeifer D, Jager E, Lubbert M (2010) The DNA demethylating agent 5-aza-2'-deoxycytidine induces expression of NY-ESO-1 and other cancer/testis antigens in myeloid leukemia cells. Leuk Res 34(7):899-905. doi:10.1016/j.leukres.2010.02.004

13. Coral S, Sigalotti L, Altomonte M, Engelsberg A, Colizzi F, Cattarossi I, Maraskovsky E, Jager E, Seliger B, Maio M (2002) 5-Aza-2'-deoxycytidine-induced expression of functional cancer testis antigens in human renal cell carcinoma: immunotherapeutic implications. Clin Cancer Res 8(8):2690-2695

14. Coral S, Sigalotti L, Gasparollo A, Cattarossi I, Visintin A, Cattelan A, Altomonte M, Maio M (1999) Prolonged upregulation of the expression of HLA class I antigens and costimulatory molecules on melanoma cells treated with 5-aza-2'-deoxycytidine (5-AZA-CdR). J Immunother 22(1):16-24

15. Rockmann H, Schadendorf D (2003) Drug resistance in human melanoma: mechanisms and therapeutic opportunities. Onkologie 26(6):581-587. doi:10.1159/000074156

16. Jazirehi AR, Baritaki S, Koya RC, Bonavida B, Economou JS (2011) Molecular mechanism of MART-1 +/A*0201 + human melanoma resistance to specific CTL-killing despite functional tumor-CTL interaction. Cancer Res 71(4):1406-1417. doi:10.1158/0008-5472.CAN-10-1296

17. Villanueva J, Vultur A, Lee JT, Somasundaram R, FukunagaKalabis M, Cipolla AK, Wubbenhorst B, Xu X, Gimotty PA, Kee D, Santiago-Walker AE, Letrero R, D'Andrea K, Pushparajan A, Hayden JE, Brown KD, Laquerre S, McArthur GA, Sosman JA, Nathanson KL, Herlyn M (2010) Acquired resistance to BRAF inhibitors mediated by a RAF kinase switch in melanoma can be overcome by cotargeting MEK and IGF-1R/PI3K. Cancer Cell 18(6):683-695. doi:10.1016/j.ccr.2010.11.023

18. Pollak M (2008) Insulin and insulin-like growth factor signalling in neoplasia. Nat Rev Cancer 8(12):915-928. doi:10.1038/nrc2536 
19. Baylin SB, Herman JG (2000) DNA hypermethylation in tumorigenesis: epigenetics joins genetics. Trends Genet: TIG 16(4):168-174

20. Plimack ER, Stewart DJ, Issa JP (2007) Combining epigenetic and cytotoxic therapy in the treatment of solid tumors. J Clin Oncol 25(29):4519-4521. doi:10.1200/JCO.2007.12.6029

21. Saunthararajah Y, Hillery CA, Lavelle D, Molokie R, Dorn L, Bressler L, Gavazova S, Chen YH, Hoffman R, DeSimone J (2003) Effects of 5-aza-2'-deoxycytidine on fetal hemoglobin levels, red cell adhesion, and hematopoietic differentiation in patients with sickle cell disease. Blood 102(12):3865-3870. doi:10.1182/blood-2003-05-1738

22. DeSimone J, Koshy M, Dorn L, Lavelle D, Bressler L, Molokie R, Talischy N (2002) Maintenance of elevated fetal hemoglobin levels by decitabine during dose interval treatment of sickle cell anemia. Blood 99(11):3905-3908

23. Issa JP, Garcia-Manero G, Giles FJ, Mannari R, Thomas D, Faderl S, Bayar E, Lyons J, Rosenfeld CS, Cortes J, Kantarjian HM (2004) Phase 1 study of low-dose prolonged exposure schedules of the hypomethylating agent 5-aza-2'-deoxycytidine (decitabine) in hematopoietic malignancies. Blood 103(5):1635-1640. doi:10.1182/blood-2003-03-0687

24. Laille E, Shi T, Garcia-Manero G, Cogle CR, Gore SD, Kumar KJ, Skikne BS, MacBeth KJ (2012) Extended dosing of oral azacitidine (CC-486) for 14 and 21 days provides more effective methylation reversal than a 7-day schedule. ASH Annual Meeting Abstracts 120(21):1337

25. Gore SD, Baylin S, Sugar E, Carraway H, Miller CB, Carducci M, Grever M, Galm O, Dauses T, Karp JE, Rudek MA, Zhao M, Smith BD, Manning J, Jiemjit A, Dover G, Mays A, Zwiebel J, Murgo A, Weng LJ, Herman JG (2006) Combined DNA methyltransferase and histone deacetylase inhibition in the treatment of myeloid neoplasms. Cancer Res 66(12):6361-6369. doi:10.1158/0008-5472.CAN-06-0080

26. Schrump DS, Fischette MR, Nguyen DM, Zhao M, Li X, Kunst TF, Hancox A, Hong JA, Chen GA, Pishchik V, Figg WD, Murgo AJ, Steinberg SM (2006) Phase I study of decitabine-mediated gene expression in patients with cancers involving the lungs, esophagus, or pleura. Clin Cancer Res 12(19):5777-5785. doi:10.1158/1078-0432.CCR-06-0669

27. Abele R, Clavel M, Dodion P, Bruntsch U, Gundersen S, Smyth J, Renard J, van Glabbeke M, Pinedo HM (1987) The EORTC early clinical trials cooperative group experience with 5-aza-2'deoxycytidine (NSC 127716) in patients with colo-rectal, head and neck, renal carcinomas and malignant melanomas. Eur $\mathrm{J}$ Cancer Clin oncol 23(12):1921-1924

28. Samlowski WE, Leachman SA, Wade M, Cassidy P, Porter-Gill P, Busby L, Wheeler R, Boucher K, Fitzpatrick F, Jones DA, Karpf AR (2005) Evaluation of a 7-day continuous intravenous infusion of decitabine: inhibition of promoter-specific and global genomic DNA methylation. J Clin Oncol 23(17):3897-3905. doi: 10.1200/JCO.2005.06.118

29. Pohlmann P, DiLeone LP, Cancella AI, Caldas AP, Dal Lago L, Campos O Jr, Monego E, Rivoire W, Schwartsmann G (2002) Phase II trial of cisplatin plus decitabine, a new DNA hypomethylating agent, in patients with advanced squamous cell carcinoma of the cervix. Am J Clin Oncol 25(5):496-501

30. Bandyopadhyay D, Mishra A, Medrano EE (2004) Overexpression of histone deacetylase 1 confers resistance to sodium butyrate-mediated apoptosis in melanoma cells through a p53-mediated pathway. Cancer Res 64(21):7706-7710. doi:10.1158/0008-5472.CAN-03-3897
31. $\mathrm{Hu} \mathrm{J}$, Colburn NH (2005) Histone deacetylase inhibition down-regulates cyclin D1 transcription by inhibiting nuclear factor- $\kappa \mathrm{B} / \mathrm{p} 65$ DNA binding. Mol Cancer Res 3(2):100-109. doi:10.1158/1541-7786.MCR-04-0070

32. Wang R, Brunner T, Zhang L, Shi Y (1998) Fungal metabolite FR901228 inhibits c-Myc and Fas ligand expression. Oncogene 17(12):1503-1508. doi:10.1038/sj.onc.1202059

33. Bhalla KN (2005) Epigenetic and chromatin modifiers as targeted therapy of hematologic malignancies. J Clin Oncol 23(17):39713993. doi:10.1200/JCO.2005.16.600

34. Lindemann RK, Gabrielli B, Johnstone RW (2004) Histonedeacetylase inhibitors for the treatment of cancer. Cell Cycle 3(6):779-788

35. Guo F, Sigua C, Tao J, Bali P, George P, Li Y, Wittmann S, Moscinski L, Atadja P, Bhalla K (2004) Cotreatment with histone deacetylase inhibitor LAQ824 enhances Apo-2L/tumor necrosis factor-related apoptosis inducing ligand-induced death inducing signaling complex activity and apoptosis of human acute leukemia cells. Cancer Res 64(7):2580-2589

36. Bali P, Pranpat M, Bradner J, Balasis M, Fiskus W, Guo F, Rocha K, Kumaraswamy S, Boyapalle S, Atadja P, Seto E, Bhalla K (2005) Inhibition of histone deacetylase 6 acetylates and disrupts the chaperone function of heat shock protein 90: a novel basis for antileukemia activity of histone deacetylase inhibitors. J Biol Chem 280(29):26729-26734. doi:10.1074/jbc.C500186200

37. Fiskus W, Pranpat M, Bali P, Balasis M, Kumaraswamy S, Boyapalle S, Rocha K, Wu J, Giles F, Manley PW, Atadja P, Bhalla $\mathrm{K}$ (2006) Combined effects of novel tyrosine kinase inhibitor AMN107 and histone deacetylase inhibitor LBH589 against BcrAbl—expressing human leukemia cells. Blood 108(2):645-652. doi:10.1182/blood-2005-11-4639

38. Richon VM, Sandhoff TW, Rifkind RA, Marks PA (2000) Histone deacetylase inhibitor selectively induces p21WAF1 expression and gene-associated histone acetylation. Proc Natl Acad Sci USA 97(18):10014-10019. doi:10.1073/pnas.180316197

39. Glaser KB, Staver MJ, Waring JF, Stender J, Ulrich RG, Davidsen SK (2003) Gene expression profiling of multiple histone deacetylase (HDAC) inhibitors: defining a common gene set produced by HDAC inhibition in T24 and MDA carcinoma cell lines. Mol Cancer Ther 2(2):151-163

40. Scuto A, Kirschbaum M, Kowolik C, Kretzner L, Juhasz A, Atadja P, Pullarkat V, Bhatia R, Forman S, Yen Y, Jove R (2008) The novel histone deacetylase inhibitor, LBH589, induces expression of DNA damage response genes and apoptosis in $\mathrm{Ph}$-acute lymphoblastic leukemia cells. Blood 111(10):50935100. doi:10.1182/blood-2007-10-117762

41. Kobayashi Y, Ohtsuki M, Murakami T, Kobayashi T, Sutheesophon K, Kitayama H, Kano Y, Kusano E, Nakagawa H, Furukawa Y (2006) Histone deacetylase inhibitor FK228 suppresses the Ras-MAP kinase signaling pathway by upregulating Rap1 and induces apoptosis in malignant melanoma. Oncogene 25(4):512524. doi:10.1038/sj.onc. 1209072

42. Steele N, Finn P, Brown R, Plumb JA (2009) Combined inhibition of DNA methylation and histone acetylation enhances gene re-expression and drug sensitivity in vivo. Br J Cancer 100(5):758-763. doi:10.1038/sj.bjc.6604932

43. Saunthararajah Y, Hillery C, Lavelle D et al (2003) Effects of 5-aza-2'-deoxycytidine on fetal hemoglobin levels, red cell adhesion, and hematopoietic differentiation in patients with sickle cell disease. Blood 102:3865-3870 